\title{
Perineal swelling in a mouse
}

Irene Ginty, BS, LATG \& Shelley Hoogstraten-Miller, DVM, MS, DACLAM

Researchers at our AAALAC-accredited animal facility use an inbred strain of mice to generate offspring for bone development studies. Animal care personnel set up a breeding unit by placing one male and one female mouse in individually ventilated microisolator cages. Each breeding pair is offered rodent chow (NIH-31; Ziegler Bros., Gardner, PA) and water ad libitum.

Animal care personnel requested a veterinarian to examine a mouse with perineal swelling. The mouse was approximately 10 weeks old and was part of a breeding unit that had failed to produce any offspring. The absence of offspring was unusual as the mice were free of murine pathogens and husbandry conditions were optimal. The light:dark cycle of the room was set at $12 \mathrm{~h}: 12 \mathrm{~h}$ and the temperature was maintained at $70{ }^{\circ} \mathrm{F} \pm 2{ }^{\circ} \mathrm{F}$.

On examination, the mouse was noted to be well-hydrated and in general good physical condition. A soft, reducible swelling was noted in the perineum (Fig. 1). Palpation of the abdomen and the perineum did not seem to cause pain to the mouse. The perineal skin was of normal color and temperature.

What gender is the mouse? What are your differential diagnoses for the perineal swelling? Could the presenting clinical sign be related to failure to produce any offspring? Is the perineal swelling treatable? If so, how would you treat it?

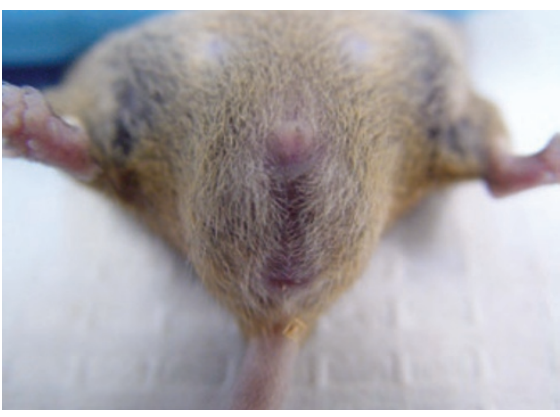

FIGURE 1 | A 10-week-old mouse with perineal swelling.

What's your diagnosis? 